\title{
Successive Convex Approximation for Transmit Power Minimization in SWIPT-Multicast Systems
}

\author{
Sumit Gautam, Eva Lagunas, Steven Kisseleff, Symeon Chatzinotas and Björn Ottersten \\ Interdisciplinary Centre for Security, Reliability and Trust (SnT), University of Luxembourg, Luxembourg. \\ Email: \{sumit.gautam, eva.lagunas, steven.kisseleff, symeon.chatzinotas, bjorn.ottersten\}@uni.lu
}

\begin{abstract}
We propose a novel technique for total transmit power minimization and optimal precoder design in wireless multi-group (MG) multicasting (MC) systems. The considered framework consists of three different systems capable of handling heterogeneous user types viz., information decoding (ID) specific users with conventional receiver architectures, energy harvesting (EH) only users with non-linear EH module, and users with joint ID and EH capabilities having separate units for the two operations, respectively. Each user is categorized under unique group(s), which can be of MC type specifically meant for ID users, and/or an energy group consisting of EH explicit users. The joint ID and EH users are a part of the (last) EH group as well as any one of the MC groups distinctly. In this regard, we formulate an optimization problem to minimize the total transmit power with optimal precoder designs for the three aforementioned scenarios, under constraints on minimum signal-to-interference-plus-noise ratio and harvested energy by the users with respective demands. The problem may be adapted to the well-known semi-definite program, which can be typically solved via relaxation of rank-1 constraint. However, the relaxation of this constraint may in some cases lead to performance degradation, which increases with the rank of the solution obtained from the relaxed problem. Hence, we develop a novel technique motivated by the feasible-point pursuit and successive convex approximation method in order to address the rank-related issue. The benefits of the proposed method are illustrated under various operating conditions and parameter values, with comparison between the three abovementioned scenarios.
\end{abstract}

\section{INTRODUCTION}

The evolution of wireless communication technologies is now happening at an even faster pace in comparison to previous generations. In this process, several critical issues like the increasing performance and capacity needs, power management at the complex hardware set-ups, and demands for energyefficient algorithms at the wireless devices, still remain great challenges to address [1]. Following these insistent demands, another challenge is posed in the form of battery limitations at the wireless devices, wherein their high power consumption leads to frequent recharging. To address this, two possibilities arise; optimization of power consumption at the devices, or seeking alternative energy harvesting $(\mathrm{EH})$ techniques for recharging [2]. The latter prospect invokes a renewed research interest leading towards investigation of systems which ensure coexistence of heterogeneous user devices, including information decoding (ID) only, specific to $\mathrm{EH}$, and ones performing ID and EH concurrently [3].

Several advantages of adopting Multiple-Input Single-Output (MISO) set-up can be seen from the perspective of ID users
[4]. An interesting idea to perform joint radio-frequency (RF) -based simultaneous wireless information and power transmission (SWIPT) was proposed in [5], which was later incorporated to the multi-user MISO case in [2], [6]. Regarding the EH modules, separated architectures (SA) seem more promising in contrast to other proposed EH models due to reduced hardware complexities and no-extra optimization parameter(s) [7]. On the other hand, transmit precoding can enhance the channel capacity and diversity in multi-user MISO systems significantly [8]. Another potential technique in this direction is termed Multi-group (MG) Multicasting (MC), where its benefits with precoding are illustrated in [9]. However, MG-MC precoding problem for only a single group multicast was found to be NP-hard in [8]. With an assumption of a linear EH module, certain works have investigated joint transmission of information and energy in an MG-MC scenario [10]. Noticeably, above mentioned works neither takes into consideration the coexistence aspect of heterogeneous user types, nor the non-linear $\mathrm{EH}$ aspect at the intended users within the MG-MC framework.

In this work, we investigate three systems with the MG-MC framework, where the goal is to minimize the total transmit power via precoder designing mechanism, in order to serve heterogeneous types of users. The single transmitter is assumed to be equipped with multiple antennas that enable the precoders to meet the demands at the intended devices via beamforming technique. Herein, the formulated optimization problem (as mentioned above) is found to be non-convex. However, it can be converted into a semi-definite programming (SDP) problem and solved using known transformations and relaxations. Even though suitable solutions are typically obtained, this method reveals a lot of uncertainties revolving around the approximations. Therefore, we propose a novel technique (for the considered MG-MC framework) as an alternative to solving the SDP, termed as Feasible Point Pursuit and Successive Convex Approximation for energy optimization (FPP-SCA-e), which is based on modified FPP-SCA [11]. The proposed technique yields significant gains both in terms of performance as well as the computational complexity. To summarize, the main contributions and novelty of this paper are highlighted below

(a) We compare three novel MG-MC precoding frameworks which deals with co-existence of three user types capable of ID, EH, and joint ID and $\mathrm{EH}$, respectively.

(b) We provide adequate transformation to reduce the nonlinear EH constraint to a linear form. Without loss of 


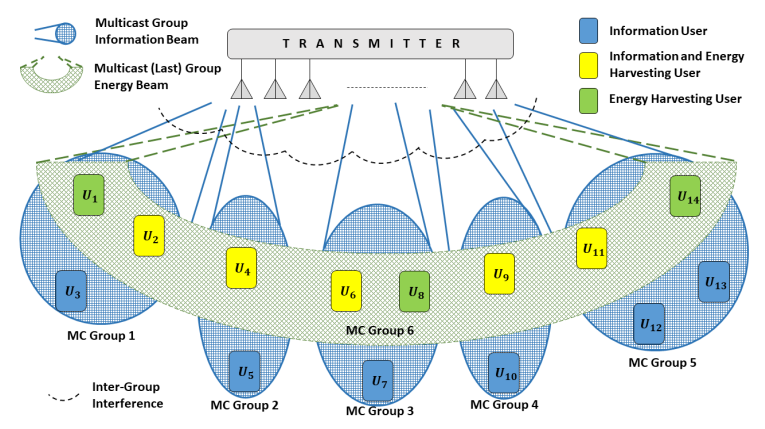

Fig. 1: Separate Multicast and Energy Precoding Design (SMEP).

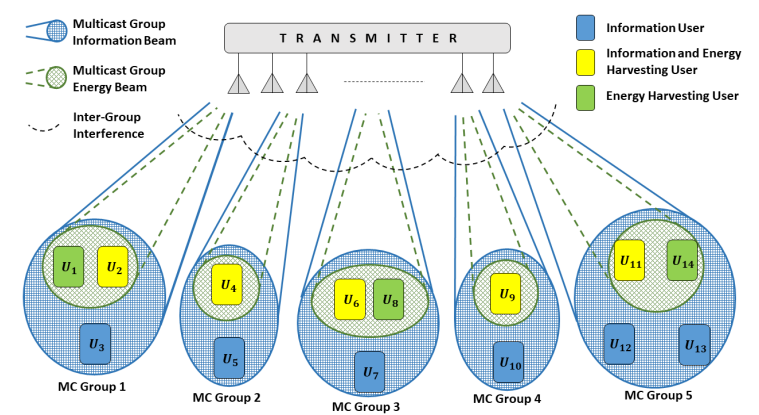

Fig. 2: Joint Multicast and Energy Precoding Design (JMEP).

generality, this transformation is useful not only for the solution of the considered problem, but potentially even more challenging problems with similar constraints.

(c) To address the limitations of SDP and the rank-concerns related to the SDR-based solutions, we propose a novel technique called FPP-SCA-e, which does not only takes into consideration the $\mathrm{EH}$ constraint in contrast to the traditional FPP-SCA method [11], [12], but also provides significant gains over the SDR-based approximations while ensuring unit rank solutions for corresponding precoder metrics.

(d) We discuss the benefits of separate information and/or energy precoder design over other designs used for comparison, to motivate its practical implementation.

The remainder of this paper is organized as follows. Section II provides an introduction to the system model. The problem formulation and the proposed solution are presented in Section III. Numerical results are shown in Section IV, followed by concluding remarks in Section V.

Notation: In the remainder of this paper, bold face lower case and upper case characters denote column vectors and matrices, respectively. The operators $(\cdot)^{\dagger},|\cdot|$ and $\otimes$ correspond to the conjugate transpose, the absolute value and the Kronecker product, respectively. An identity matrix of $Y \times Y$ dimensions is denoted as $\mathbf{I}_{Y}$, where its $y^{\text {th }}$ column is represented as $\mathbf{e}_{y}$. Calligraphic indexed characters denote sets. $\mathbb{R}_{\kappa}^{+}$denotes the set of real positive $\kappa$-dimensional vectors.

\section{SySTEM MODEL}

In this work, we assume that $K$ users $\left(\mathcal{U}_{1}, \ldots, \mathcal{U}_{K}\right)$ are served via single transmit source equipped with $M$ antennas. The heterogeneous receiver types, viz-a-viz., ID, EH, and joint

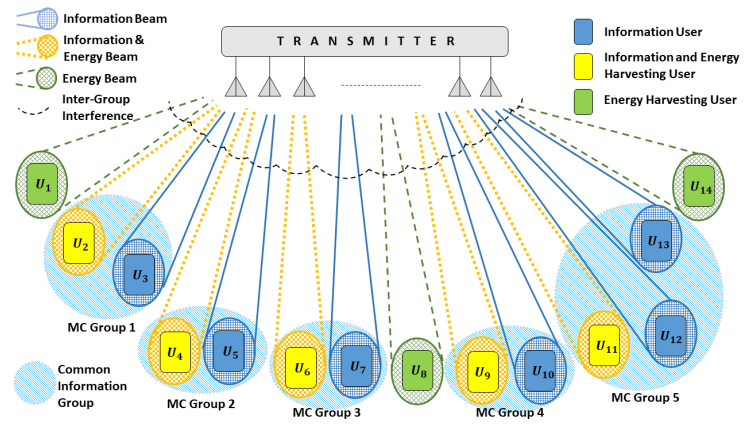

Fig. 3: Per-User Information and/or Energy Precoding Design (PIEP).

ID and $\mathrm{EH}$ are categorized within $(Z+1)$ groups. In the case with both ID and EH operations, corresponding user adopts the separated architecture (SA) for SWIPT [7], with two separate RF chains for carrying out the desired operations.

We examine three precoder design schemes for optimization of total transmit power, described as follows.

(a) Separate Multicast and Energy Precoding Design (SMEP): We aim at designing $(Z+1)$ precoders, where $Z$ precoders are designated to serve $Z$ MC groups and an additional precoder exclusively takes care of $(Z+1)^{\text {th }}$ group with EH users. (Ref. Fig. 1)

(b) Joint Multicast and Energy Precoding Design (JMEP): We target the design of $Z$ precoders for fulfilling the ID and $\mathrm{EH}$ demands of respective $Z$ multicast precoders and the additional group with EH users. Specifically, there is no specific precoder for serving EH users. (Ref. Fig. 2)

(c) Per-User Information and/or Energy Precoding Design (PIEP): Herein, design of $K$ precoders (equal to the number of users) is intended, where each user is served by its dedicated precoder. (Ref. Fig. 3)

We assume $Z$ MC groups and the $(Z+1)^{\text {th }}$ group with $\mathrm{EH}$ users, where all the classifications are already known. In this context, we define the following variable to assist the precoder design metrics for three scenarios interchangeably

$$
\Psi=\left\{\begin{array}{ccl}
Z+1 & : & \mathrm{SMEP} \rightarrow \psi(a) . \\
Z & : & \mathrm{JMEP} \rightarrow \psi(b) . \\
K & : & \operatorname{PIEP} \rightarrow \psi(c) .
\end{array}\right.
$$

Let $\mathcal{Z}_{k}$ denote the $k^{\text {th }}$ multicast/energy group of users such that $\mathcal{Z}_{k} \cap \mathcal{Z}_{\ell}=\varnothing, \forall k, \ell=\{1, \ldots, \Psi\}$ and $k \neq \ell$; whereas in case of $\mathrm{EH}$, the user harvests energy using all the possible multicast signals ${ }^{1}$.

Define $\mathbf{w}_{k}$ as the related $M \times 1$ complex precoder weight vector to serve the ID and/or $\mathrm{EH}$ user(s) corresponding to $\mathcal{Z}_{k}$. The transmitter emits the signal $\mathbf{x}(t)=\sum_{k=1}^{\Psi} \mathbf{w}_{k} s_{k}(t)$, where $s_{k}(t)$ is the corresponding information and/or energy signal. The signals for each group $\left\{s_{k}(t)\right\}_{k=1}^{\Psi}$ are mutually uncorrelated to each other with zero mean and unit variance. Distinct ID and EH signal waveform designing [13] motivates the use of SA-based SWIPT receiver architecture. The total transmit power is represented as $\sum_{k=1}^{\Psi} \mathbf{w}_{k}^{\dagger} \mathbf{w}_{k}$.

\footnotetext{
${ }^{1}$ The other MCs are primarily taken into consideration due to interference causing side-lobes other than the desired MC, which is beneficial for $\mathrm{EH}$.
} 
The signal received at the $i^{\text {th }}$ user is given by $u_{i}(t)=$ $\mathbf{h}_{i}^{\dagger} \mathbf{x}(t)+n_{R, i}(t)$, where $\mathbf{h}_{i}$ is the $M \times 1$ conjugated channel vector for the corresponding user and $n_{R, i}(t)$ is the additive zero mean Gaussian noise at the corresponding $i^{\text {th }}$ user's receiving antenna equipment with a noise variance of $\sigma_{R, i}^{2}$. The source signals are uncorrelated with $n_{R, i}(t)$. The signal at the ID module of $i^{\text {th }}$ receiver equipment is expressed as

$$
u_{D, i}(t)=\left(\mathbf{h}_{i}^{\dagger} \mathbf{x}(t)+n_{R, i}(t)\right)+n_{D, i}(t)
$$

where $n_{D, i}(t)$ is the additional zero-mean Gaussian noise with a variance of $\sigma_{D, i}^{2}$ which mainly arise due to the circuitry associated with the ID block of the $i^{\text {th }}$ receiver. The signalto-interference-plus-noise ratio (SINR) at the $i^{\text {th }}$ receiver as a part of the $k^{\text {th }}$ multicast group $\mathcal{Z}_{k}$, is given by

$$
\Upsilon_{i}=\frac{\left|\mathbf{w}_{k}^{\dagger} \mathbf{h}_{i}\right|^{2}}{\sum_{\ell \neq k}\left|\mathbf{w}_{\ell}^{\dagger} \mathbf{h}_{i}\right|^{2}+\sigma_{R, i}^{2}+\sigma_{D, i}^{2}}, \forall \ell=\{1, \ldots, \Psi\} .
$$

The signal utilized for $\mathrm{EH}$ operation at the $i^{\text {th }}$ receiver is

$$
u_{E, i}(t)=\mathbf{h}_{i}^{\dagger} \mathbf{x}(t)+n_{R, i}(t)
$$

Therefore, the energy extracted by the $\mathrm{EH}$ unit of $i^{\text {th }}$ receiver is given as, $\mathcal{E}_{i}^{\mathcal{L}}=\zeta_{i}\left(\sum_{k=1}^{\Psi}\left|\mathbf{w}_{k}^{\dagger} \mathbf{h}_{i}\right|^{2}+\sigma_{R, i}^{2}\right)$, where $0<\zeta_{i} \leq$ 1 is the energy conversion efficiency of the EH unit at the corresponding receiver. Note that $\mathcal{E}_{i}^{\mathcal{L}}$ is theoretically valid in order to represent a linear $\mathrm{EH}$ operation, however its practical implementation is doubtful. Thus, this calls for the adoption of a non-linear EH model [14] at the $i^{\text {th }}$ receiver, defined as

$$
\mathcal{E}_{i}^{\mathcal{N}}=\frac{\mathcal{E}^{\prime}}{1-\phi} \cdot\left(\frac{1}{1+e^{\left(-\alpha\left(\sum_{k=1}^{\Psi}\left|\mathbf{w}_{k}^{\dagger} \mathbf{h}_{i}\right|^{2}\right)+\alpha \beta\right)}}-\phi\right),
$$

where $\phi \triangleq \frac{1}{1+\exp (\alpha \beta)}$, the constant $\mathcal{E}^{\prime}$ is obtained by determining the maximum harvested energy on the saturation of the energy harvesting circuit, and $\alpha$ and $\beta$ are specific for the capacitor and diode turn-on voltage metrics at the EH circuit. Practically, a standard curve-fitting tool based on analytical data may be used to decide the appropriate values of $\mathcal{E}^{\prime}, \alpha$, and $\beta$.

We assume that a central controller has access to channel state information (CSI) and the user demands and informs the concerned nodes about the resulting optimization through a separate channel and appropriate signaling. Additionally, we consider normalized time slots to use the terms power and energy interchangeably.

In the succeeding section, we formulate an optimization problem corresponding to precoder designs for minimization of the total transmit power in the three aforementioned scenarios. Suitable solutions are obtained by employing adequate transformations and relaxations.

\section{TRAnsmit Power Minimization}

In this section, we formulate the optimization problem to minimize the total transmit power subjected to minimum SINR and minimum (non-linear) EH constraints at the corresponding users/groups.

\section{A. Problem Formulation}

The overall optimization problem (encapsulating the three considered scenarios) to ensure the co-existence of the het- erogeneous user types in the MG-MC precoding scheme can subsequently be written in its analytical form as follows

$$
\begin{aligned}
(P 1): \min _{\left\{\mathbf{w}_{k}\right\}_{k=1}^{\Psi}} & \sum_{k=1}^{\Psi} \mathbf{w}_{k}^{\dagger} \mathbf{w}_{k} \\
\text { s.t. } & (C 1): \frac{\left|\mathbf{w}_{k}^{\dagger} \mathbf{h}_{i}\right|^{2}}{\sum_{\ell \neq k}\left|\mathbf{w}_{\ell}^{\dagger} \mathbf{h}_{i}\right|^{2}+\sigma_{R, i}^{2}+\sigma_{D, i}^{2}} \geq \gamma_{i}, \\
& \forall \Xi[(i, k, \ell) \mid \psi], \\
& (C 2): \mathcal{E}_{j}^{\mathcal{N}} \geq \xi_{j}, \forall j \in \mathcal{Z}_{Z+1},
\end{aligned}
$$

where $\gamma_{i}$ is the SINR threshold at the $i^{\text {th }}$ user, and $\xi_{j}$ is the demanded harvested energy at $j^{\text {th }}$ user (where $i$ can be equal to $j$ for some cases, in general). We define the following metric to assist $(C 1)$, as a conditional indicator for the three scenarios

$$
\Xi[(i, k, \ell) \mid \psi]=\left\{\begin{aligned}
\psi(a): & \forall i \in \mathcal{Z}_{k}, \forall k \in\{1, \ldots, Z\}, \\
& \forall \ell \in\{1, \ldots, Z+1\}, \\
\psi(b): & \forall i \in \mathcal{Z}_{k}, \forall k \in\{1, \ldots, Z\}, \\
& \forall \ell \in\{1, \ldots, Z\}, \\
\psi(c): & \forall i, \forall k, \forall \ell \in\{1, \ldots, K\},
\end{aligned}\right.
$$

where $\psi(a), \psi(b)$, and $\psi(c)$ represents the SMEP, JMEP and PIEP scenarios, respectively. The indicator metric $\Xi[(i, k, \ell) \mid \psi]$ implies that the selections of $(i, k, \ell)$ are conditional according to the chosen scenario, i.e., $\psi$, where $\psi$ corresponds to $\psi(a)$, and/or $\psi(b)$ and/or $\psi(c)$.

It is clear that the formulated problem $(P 1)$ is not convex due to constraints $(C 1)$ and $(C 2)$ where its feasibility is dependent on $\gamma_{i}$ and $\xi_{i}$, respectively. Moreover, it is interesting to note the hidden linearity aspect within the non-linear $\mathrm{EH}$ expression in (5), which can be useful in converting the non-linear EH constraint to a linear form, without loss of generality. The corresponding transformation is provided in Appendix A of [3], [15]. In order to carry out further analysis, we simplify problem $(P 1)$ without loss of generality, as follows

$$
\begin{aligned}
(P 2): \min _{r,\left\{\mathbf{w}_{k}\right\}_{k=1}^{\Psi}} r & \\
\text { s.t. }(C 1) & : \frac{\left|\mathbf{w}_{k}^{\dagger} \mathbf{h}_{i}\right|^{2}}{\sum_{\ell \neq k}\left|\mathbf{w}_{\ell}^{\dagger} \mathbf{h}_{i}\right|^{2}+\sigma_{R, i}^{2}+\sigma_{D, i}^{2}} \geq \gamma_{i}, \\
\forall & \Xi(i, k, \ell) \mid \psi] \\
(C 2) & : \zeta_{j}\left(\sum_{k=1}^{\Psi}\left|\mathbf{w}_{k}^{\dagger} \mathbf{h}_{i}\right|^{2}+\sigma_{R, j}^{2}\right) \geq \xi_{j}^{\prime} \\
\forall j & \in \mathcal{Z}_{Z+1}, \\
(C 3) & : \sum_{k=1}^{\Psi} \mathbf{w}_{k}^{\dagger} \mathbf{w}_{k} \leq r
\end{aligned}
$$

where $r \in \mathbb{R}^{+}$is the introduced slack variable to provide more tractability to the problem, and $\xi_{j}^{\prime}$ is the modified harvested energy demand at $j^{\text {th }}$ user. Note that (C2) is a linear constraint introduced to simplify the problem. Proof for the corresponding transformation is provided in Appendix A of [3], [15]. The introduction of the slack-variable $r$, constraints 
the total transmit power. Subsequently, at the optimum $r^{\star}$, the overall transmit power is minimized. Similar to $(P 1)$, the problem $(P 2)$ is non-convex. However, its formulation is more suitable for the proposed optimization method [12]. Therefore, we further investigate the problem in the succeeding sections.

\section{B. Semi-Definite Programming (SDP) with Relaxation and Gaussian Randomization}

We define $\mathbf{H}_{i}=\mathbf{h}_{i} \mathbf{h}_{i}^{\dagger}, \mathbf{w}=\left[\begin{array}{lll}\mathbf{w}_{1}^{\mathrm{T}} & \mathbf{w}_{2}^{\mathrm{T}} \ldots \mathbf{w}_{\Psi}^{\mathrm{T}}\end{array}\right]^{\mathrm{T}}$ and $\mathbf{W}_{k}=$ $\mathbf{w}_{k} \mathbf{w}_{k}^{\dagger}, \forall k=\{1, \ldots, \Psi\}$. Then, with the help of these notations, $(P 2)$ can be molded into a semi-definite programming (SDP) problem, where the non-convexity of $(P 2)$ lies in the necessity to constrain the variable $\mathbf{W}_{k}$ to have unit rank. By dropping the rank-1 constraint, we obtain the following relaxed problem

$$
\begin{aligned}
(P 3): \min _{r,\left\{\mathbf{W}_{k}\right\}_{k=1}^{\Psi}} r \\
\text { s.t. }(C 1): \operatorname{Tr}\left\{\mathbf{H}_{i} \mathbf{W}_{k}\right\}-\gamma_{i} \sum_{\ell \neq k} \operatorname{Tr}\left\{\mathbf{H}_{i} \mathbf{W}_{\ell}\right\} \\
\geq \gamma_{i}\left(\sigma_{R, i}^{2}+\sigma_{D, i}^{2}\right), \\
\forall \Xi[(i, k, \ell) \mid \psi], \\
(C 2): \sum_{k=1}^{\Psi} \operatorname{Tr}\left\{\mathbf{H}_{j} \mathbf{W}_{k}\right\} \geq \frac{\xi_{j}^{\prime}}{\zeta_{j}}-\sigma_{R, j}^{2}, \\
\forall j \in \mathcal{Z}_{Z+1}, \\
(C 3): \sum_{k=1}^{\Psi} \operatorname{Tr}\left\{\mathbf{W}_{k}\right\} \leq r, \\
(C 4): \mathbf{W}_{k} \succcurlyeq 0 .
\end{aligned}
$$

The SDP problem in $(P 3)$ is convex and can be solved via wellknown convex optimization techniques [16]. For our numerical evaluations, we make use of the convex programming tool CVX [17]. Following the semi-definite relaxation (SDR) in $(P 3)$, the derivation of optimal $\mathbf{w}_{k}^{\star}$ requires a rank-1 approximation over $\mathbf{W}_{k}^{\star}$. This approximation may be obtained by using the Eigen-value Decomposition (EVD) [18] of $\mathbf{W}_{k}^{\star}$ and selecting the eigen-vector, which pertains to the maximum eigen-value. In this context, it is explicit that if $\operatorname{rank}\left(\mathbf{W}_{k}^{\star}\right)=1, \forall k$, then the obtained solutions are indeed optimal. However, the analytical results indicate towards the presence of multi-rank solutions corresponding to the precoder serving the EH group of users via SMEP, while unit rank solutions are obtained for all the other cases and scenarios. In this regard, Gaussian randomization method ${ }^{2}$ [8] is employed to the former (i.e., precoder metric corresponding to the $\mathrm{EH}$ group of users) due to its high accuracy in yielding a rank-1 approximation of $\left\{\mathbf{W}_{Z+1}\right\}$ (in case of SMEP). In this context, SDR method with randomization is proven to be an effective and lowcomplexity approximation technique [12]. However, alternative ways may be explored to further improve the approximation of the solution. In the succeeding section, we present a novel

\footnotetext{
${ }^{2}$ Based on the statistics outlined by the relaxed solution, precoding vectors are generated with the help of Gaussian distribution. The solution nearest to the relaxed upper bound is chosen after creating several re-scaled instances.
}

technique to address the multi-rank issue and provide better solutions in comparison with the SDR technique.

\section{Novel Feasible Point Pursuit and Successive Convex Ap- proximation Method for Energy Optimization (FPP-SCA-e)}

In this section, we present an enhanced technique motivated by a recently proposed alternative to SDR, namely, feasiblepoint pursuit and successive convex approximation (FPP-SCA) [11], [12]. In contrast to the traditional FPP-SCA method applied to such frameworks [12], the technique proposed in this paper, FPP-SCA-e, takes care of an additional constraint of harvested energy demand at the intended users. It is clear that the problem $(P 2)$ can be categorized within the general class of quadratically constrained quadratic problems (QCQPs), and thus a modified technique in-line with FPP-SCA can be developed which also takes into account an additional harvested energy constraint. To proceed, we define $\mathbf{w}_{\text {tot }}=$ $\left[\mathbf{w}_{1}^{\dagger}, \mathbf{w}_{2}^{\dagger}, \ldots, \mathbf{w}_{\Psi}^{\dagger}\right]^{\dagger}$ such that the $i^{\text {th }}$ SINR constraint reads as

$$
\mathbf{w}_{\mathrm{tot}}^{\dagger} \boldsymbol{\Lambda}_{i} \mathbf{w}_{\mathrm{tot}} \leq-\gamma_{i}\left(\sigma_{R, i}^{2}+\sigma_{D, i}^{2}\right)
$$

where $\boldsymbol{\Lambda}_{i}=\boldsymbol{\Lambda}_{i}^{(+)}+\boldsymbol{\Lambda}_{i}^{(-)}$with $\boldsymbol{\Lambda}_{i}^{(+)}=\gamma_{i}\left(\mathbf{I}_{\Psi}-\operatorname{diag}\left\{\mathbf{e}_{k}\right\}\right) \otimes$ $\mathbf{h}_{i} \mathbf{h}_{i}^{\dagger}, \quad \Lambda_{i}^{(-)}=-\operatorname{diag}\left\{\mathbf{e}_{k}\right\} \otimes \mathbf{h}_{i} \mathbf{h}_{i}^{\dagger}, \forall i \in \mathcal{Z}_{k}$, with $k \in$ $\{1, \ldots, \Psi\}$. Let $\boldsymbol{\omega}$ denote any random point so that by the definition of a semi-definite matrix $\Lambda_{i}^{(-)}$we have ( $\mathbf{w}_{\text {tot }}-$ $\boldsymbol{\omega})^{\dagger} \boldsymbol{\Lambda}_{i}^{(-)}\left(\mathbf{w}_{\text {tot }}-\boldsymbol{\omega}\right) \leq 0$. With further simplification, we express the linear constraint of $\mathbf{w}_{\text {tot }}$ around $\boldsymbol{\omega}$ as follows

$$
\mathbf{w}_{\text {tot }}^{\dagger} \Lambda_{i}^{(-)} \mathbf{w}_{\text {tot }} \leq 2 \operatorname{Re}\left\{\boldsymbol{\omega}^{\dagger} \boldsymbol{\Lambda}_{i}^{(-)} \mathbf{w}_{\text {tot }}\right\}-\boldsymbol{\omega}^{\dagger} \boldsymbol{\Lambda}_{i}^{(-)} \boldsymbol{\omega} .
$$

Subsequently, the SINR constraint in (20) is given by

$$
\begin{aligned}
\mathbf{w}_{\text {tot }}^{\dagger} \boldsymbol{\Lambda}_{i}^{(+)} \mathbf{w}_{\text {tot }}+2 \operatorname{Re} & \left\{\boldsymbol{\omega}^{\dagger} \boldsymbol{\Lambda}_{i}^{(-)} \mathbf{w}_{\text {tot }}\right\} \\
& -\boldsymbol{\omega}^{\dagger} \boldsymbol{\Lambda}_{i}^{(-)} \boldsymbol{\omega} \leq-\gamma_{i}\left(\sigma_{R, i}^{2}+\sigma_{D, i}^{2}\right),
\end{aligned}
$$

wherein the unknown variables are quadratic over a semidefinite matrix. Following a similar trend, the harvested energy constraint at the $\epsilon^{\text {th }}$ user can thus be represented as

$$
\begin{aligned}
\zeta_{\epsilon}\left[\boldsymbol{\omega}^{(j) H} \boldsymbol{\Lambda}_{\epsilon}^{(-)} \boldsymbol{\omega}^{(j)}-2\right. & \operatorname{Re}\left\{\boldsymbol{\omega}^{(j) H} \boldsymbol{\Lambda}_{\epsilon}^{(-)} \mathbf{w}_{\mathrm{tot}}\right\} \\
& \left.-\mathbf{w}_{\mathrm{tot}}^{\dagger} \hat{\boldsymbol{\Lambda}}_{\epsilon}^{(+)} \mathbf{w}_{\mathrm{tot}}+\sigma_{R, \epsilon}^{2}\right] \geq \xi_{\epsilon}^{\prime},
\end{aligned}
$$

where $\hat{\boldsymbol{\Lambda}}_{\epsilon}^{(+)}=\left(\mathrm{I}_{\Psi}-\operatorname{diag}\left\{\mathbf{e}_{k}\right\}\right) \otimes \mathbf{h}_{\epsilon} \mathbf{h}_{\epsilon}^{\dagger}, \forall \epsilon \in \mathcal{Z}_{Z+1}$, with $k \in\{1, \ldots, \Psi\}$. By adding the slack penalties $\mathbf{v} \in \mathbb{R}_{(Z+2)}^{+}$, the QCQP problem in $(P 2)$ can be approximated as follows

$$
\begin{array}{rl}
(P 4): \min _{r, \mathbf{w}_{\text {tot }, \mathbf{v}}} & r+\lambda\|\mathbf{v}\| \\
\text { s.t. } & (C 1): \mathbf{w}_{\mathrm{tot}}^{\dagger} \boldsymbol{\Lambda}_{i}^{(+)} \mathbf{w}_{\mathrm{tot}}+2 \operatorname{Re}\left\{\boldsymbol{\omega}^{(j) \dagger} \boldsymbol{\Lambda}_{i}^{(-)} \mathbf{w}_{\text {tot }}\right\} \\
-\boldsymbol{\omega}^{(j) \dagger} \boldsymbol{\Lambda}_{i}^{(-)} \boldsymbol{\omega}^{(j)} \leq-\gamma_{i}\left(\sigma_{R, i}^{2}+\sigma_{D, i}^{2}\right)+\mathbf{v}_{\mathcal{I}}, \\
\forall \Xi[(i, k, \ell) \mid \psi], \mathcal{I} \in\{1, \ldots, Z\} \\
(C 2): \zeta_{\epsilon}\left[\boldsymbol{\omega}^{(j) \dagger} \boldsymbol{\Lambda}_{\epsilon}^{(-)} \boldsymbol{\omega}^{(j)}-2 \operatorname{Re}\left\{\boldsymbol{\omega}^{(j) \dagger} \boldsymbol{\Lambda}_{\epsilon}^{(-)} \mathbf{w}_{\text {tot }}\right\}\right. \\
\left.-\mathbf{w}_{\mathrm{tot}}^{\dagger} \hat{\boldsymbol{\Lambda}}_{\epsilon}^{(+)} \mathbf{w}_{\text {tot }}+\sigma_{R, \epsilon}^{2}\right] \geq \xi_{\epsilon}^{\prime}-\mathrm{v}_{Z+1} \\
\forall \epsilon \in \mathcal{Z}_{Z+1} \\
(C 3): \mathbf{w}_{\mathrm{tot}}^{\dagger} \mathbf{w}_{\text {tot }} \leq r+\mathbf{v}_{Z+2},
\end{array}
$$




\begin{tabular}{|c|c|c|c|c|c|c|c|c|c|c|}
\hline \multicolumn{2}{|c|}{ Technique $\rightarrow$} & \multicolumn{3}{|c|}{ JMEP } & \multicolumn{3}{|c|}{ SMEP } & \multicolumn{3}{|c|}{ PIEP } \\
\hline Antennas $\downarrow$ & $\left(\gamma_{i}, \xi_{i}\right) \downarrow$ & SDP & $\begin{array}{l}\text { FPP- } \\
\text { SCA-e }\end{array}$ & $\begin{array}{c}\text { FPP- } \\
\text { SCA-e }^{+}\end{array}$ & SDP & $\begin{array}{l}\text { FPP- } \\
\text { SCA-e }\end{array}$ & $\begin{array}{c}\text { FPP- } \\
\text { SCA-e }{ }^{+}\end{array}$ & SDP & $\begin{array}{l}\text { FPP- } \\
\text { SCA-e }\end{array}$ & $\begin{array}{c}\text { FPP- } \\
\text { SCA-e }{ }^{+}\end{array}$ \\
\hline \multirow{5}{*}{$M=20$} & $(0.1 \mathrm{~dB}, 1 \mu \mathrm{J})$ & 25.4 & 5.6 & 31.0 & 43.7 & 6.9 & 50.4 & 268.8 & 15.9 & 284.0 \\
\hline & $(25 \mathrm{~dB}, 1 \mu \mathrm{J})$ & 31.5 & 6.0 & 37.0 & 55.5 & 6.9 & 62.4 & 354.0 & 17.0 & 366.5 \\
\hline & $(0.1 \mathrm{~dB}, 5 \mu \mathrm{J})$ & 25.4 & 5.6 & 31.0 & 47.7 & 6.8 & 55.0 & 275.6 & 16.8 & 288.0 \\
\hline & $(25 \mathrm{~dB}, 5 \mu \mathrm{J})$ & 29.8 & 6.1 & 35.3 & 55.4 & 6.9 & 62.1 & 373.7 & 16.3 & 389.0 \\
\hline & AVERAGE & 28.025 & 5.825 & 33.575 & 50.575 & 6.875 & 57.475 & 318.025 & 16.500 & 331.875 \\
\hline \multirow{5}{*}{$M=10$} & $(0.1 \mathrm{~dB}, 1 \mu \mathrm{J})$ & 2.4 & 5.3 & 7.6 & 2.7 & 6.5 & 9.3 & 4.9 & 14.6 & 17.8 \\
\hline & $(25 \mathrm{~dB}, 1 \mu \mathrm{J})$ & 2.6 & 5.4 & 7.8 & 3.6 & 6.6 & 10.2 & 6.6 & 14.8 & 18.4 \\
\hline & $(0.1 \mathrm{~dB}, 5 \mu \mathrm{J})$ & 2.4 & 5.3 & 7.8 & 2.8 & 6.4 & 9.4 & 5.9 & 14.8 & 18.5 \\
\hline & $(25 \mathrm{~dB}, 5 \mu \mathrm{J})$ & 2.9 & 5.7 & 8.2 & 3.5 & 6.5 & 10.0 & 7.6 & 14.9 & 20.4 \\
\hline & AVERAGE & 2.575 & 5.425 & 7.850 & 3.150 & 6.500 & 9.725 & 6.250 & 14.775 & 18.775 \\
\hline
\end{tabular}

TABLE I: Computational complexity analysis (in seconds) of the proposed methods.

\begin{tabular}{|c|c|c|c|}
\hline$\xi_{i}$ Technique & SDP & FPP-SCA-e & FPP-SCA-e $^{+}$ \\
\hline $1 \mu \mathrm{J}$ & $10.6347 \mathrm{dBW}$ & $10.1224 \mathrm{dBW}$ & $9.4784 \mathrm{dBW}$ \\
\hline $2 \mu \mathrm{J}$ & $13.6151 \mathrm{dBW}$ & $12.8914 \mathrm{dBW}$ & $12.4496 \mathrm{dBW}$ \\
\hline $3 \mu \mathrm{J}$ & $15.3523 \mathrm{dBW}$ & $14.7874 \mathrm{dBW}$ & $14.0671 \mathrm{dBW}$ \\
\hline $4 \mu \mathrm{J}$ & $16.5805 \mathrm{dBW}$ & $15.7903 \mathrm{dBW}$ & $15.5425 \mathrm{dBW}$ \\
\hline $5 \mu \mathrm{J}$ & $17.5288 \mathrm{dBW}$ & $16.8827 \mathrm{dBW}$ & $16.4088 \mathrm{dBW}$ \\
\hline
\end{tabular}

TABLE II: Total Transmit Power for SMEP, optimized using SDP, SCA, and FPP-SCA-e ${ }^{+}$schemes, with $\gamma_{i}=5 \mathrm{~dB}$ and $M=16$.

where $r \in \mathbb{R}^{+}, \lambda \in \mathbb{R}$ is a fixed input parameter and $\boldsymbol{\omega}^{(j)}$ is the $j^{\text {th }}$ instance of the introduced auxiliary variable. Herein, $(P 4)$ is a convex QCQP and can be solved via well-known convex optimization techniques [16]. Similar to FPP-SCA as in [11], $(P 4)$ is solved with starting point selection as $\boldsymbol{\omega}^{(j+1)}=\mathbf{w}_{\text {tot }}^{(j)}$ in each instance of the FPP-SCA-e algorithm. This iterative process is repeated until guaranteed convergence [11], [12]. The convergence proof is straight-forward. Since the modification to the problem is treated in the same way, i.e., eigenvalue separation; there is no change in the behavior of FPP-SCAe technique with respect to the original FPP-SCA.

\section{NuMERICAL RESUlts}

In this section, we present the performance benefits of the proposed FPP-SCA-e over SDR, both employed on the three considered scenarios, viz., SMEP, JMEP, and PIEP. All these models and techniques are implemented using MATLAB R2017a, with optimization performed via convex programming tool CVX [17], and the solutions obtained with the help of SEDUMI solver.

\section{A. Simulation Set-up}

Herein, the path-loss exponent for generating the channel realizations is chosen according to the ITU-R indoor model (2-floor office scenario) [19] as follows

$$
\mathrm{PL}(\text { in } \mathrm{dB})=20 \log _{10}(F)+N \log _{10}(D)+P_{f}(n)-28,
$$

where $F$ is the operational frequency (in $\mathrm{MHz}$ ), $N$ is the distance power loss coefficient, $D$ is the separation distance (in metres) between the transmitter and end-user(s) (with $D>1 \mathrm{~m}$ ), $P_{f}(n)=15+4(n-1):$ is the floor penetration loss factor (in $\mathrm{dB})$, and $n$ is the number of floors between the transmitter and the end-user(s) (with $n \geq 0$ ). Specifically, the chosen parametric values are : $F=2.4 \mathrm{GHz}, D=5 \mathrm{~m}$ (unless specified otherwise),

\begin{tabular}{|c|c|c|c|}
\hline $\begin{array}{ll} & \text { Technique } \\
\xi_{i} & \end{array}$ & SDP & FPP-SCA-e & FPP-SCA-e ${ }^{+}$ \\
\hline $1 \mu \mathrm{J}$ & $10.6347 \mathrm{dBW}$ & $9.8102 \mathrm{dBW}$ & $9.2665 \mathrm{dBW}$ \\
\hline $2 \mu \mathrm{J}$ & $13.6152 \mathrm{dBW}$ & $12.6895 \mathrm{dBW}$ & $12.3341 \mathrm{dBW}$ \\
\hline $3 \mu \mathrm{J}$ & $15.3527 \mathrm{dBW}$ & $14.8355 \mathrm{dBW}$ & $14.0506 \mathrm{dBW}$ \\
\hline $4 \mu \mathrm{J}$ & $16.5805 \mathrm{dBW}$ & $15.7481 \mathrm{dBW}$ & $15.4584 \mathrm{dBW}$ \\
\hline $5 \mu \mathrm{J}$ & $17.5288 \mathrm{dBW}$ & $16.7515 \mathrm{dBW}$ & $16.3696 \mathrm{dBW}$ \\
\hline
\end{tabular}

TABLE III: Total Transmit Power for JMEP, optimized using SDP, SCA, and FPP-SCA-e ${ }^{+}$schemes, with $\gamma_{i}=5 \mathrm{~dB}$ and $M=16$.

$N=30$, and $P_{f}(2)=19 \mathrm{~dB}$. The transmitter is assumed to be equipped with $M=20$ antennas (unless specified otherwise) while $K=10$ users are distributed within $(Z+1)=5$ groups as follows: $\mathcal{Z}_{1}=\left\{U_{1}, U_{3}, U_{4}\right\}, \mathcal{Z}_{2}=\left\{U_{2}, U_{5}\right\}, \mathcal{Z}_{3}=\left\{U_{6}, U_{8}\right\}$, $\mathcal{Z}_{4}=\left\{U_{7}, U_{9}, U_{10}\right\}$, and $\mathcal{Z}_{5}=\left\{U_{1}, U_{5}, U_{8}, U_{10}\right\}$, where $\mathcal{Z}_{5}$ is the EH group of users while the remaining $\left(\mathcal{Z}_{1}, \cdots, \mathcal{Z}_{4}\right) \mathrm{MC}$ groups are comprised of ID users. We set to $\sigma_{R, i}^{2}=-110 \mathrm{dBW}$, $\sigma_{D, i}^{2}=-80 \mathrm{dBW}$ and $\zeta_{i}=0.6$. Furthermore, an average of 500 random channel realizations (with random placement of endusers in every realization) is presented for each experiment. The constants for EH circuit are chosen as $\mathcal{E}^{\prime}=2.8 \mathrm{~mJ}, \alpha=$ 1500 , and $\beta=0.0022$ [14], [20].

\section{B. Analysis of the FPP-SCA-e Solutions}

Firstly, we focus on two different possibilities related to the initial point selection for $\omega$ in the proposed method corresponding to $(P 4)$. Let the starting point of $\boldsymbol{\omega}$ be denoted by $\widehat{\mathbf{w}}_{\text {tot }}$. The first possibility considers any random selection of $\widehat{\mathbf{w}}_{\text {tot }}$, which we term as FPP-SCA-e. Whereas for the second possibility, we provide the solution obtained via SDR as the input to $\widehat{\mathbf{w}}_{\text {tot }}$, which we refer to as FPP-SCA-e ${ }^{+}$.

Next, we consider the computational complexities of the proposed techniques, wherein we present their run-time analysis in Table I using the tic-toc function in MATLAB R2017a, with all the reported values in seconds. On one hand, we observe that the execution time of SDP is faster than FPP-SCA-e for lower number of transmit antennas, in all the three scenarios. While on the other hand, significant run-time increment is seen in the case of SDP over FPP-SCA-e, for high number of transmit antennas. The FPP-SCA- $\mathrm{e}^{+}$technique relies on the starting point of SDP and thus behaves according to the execution times of both SDP and FPP-SCA-e. Specifically, the increased run-time for SDP ranges approximately between $150 \%$ and $200 \%$ (depending on the type of scheme chosen), whereas the 


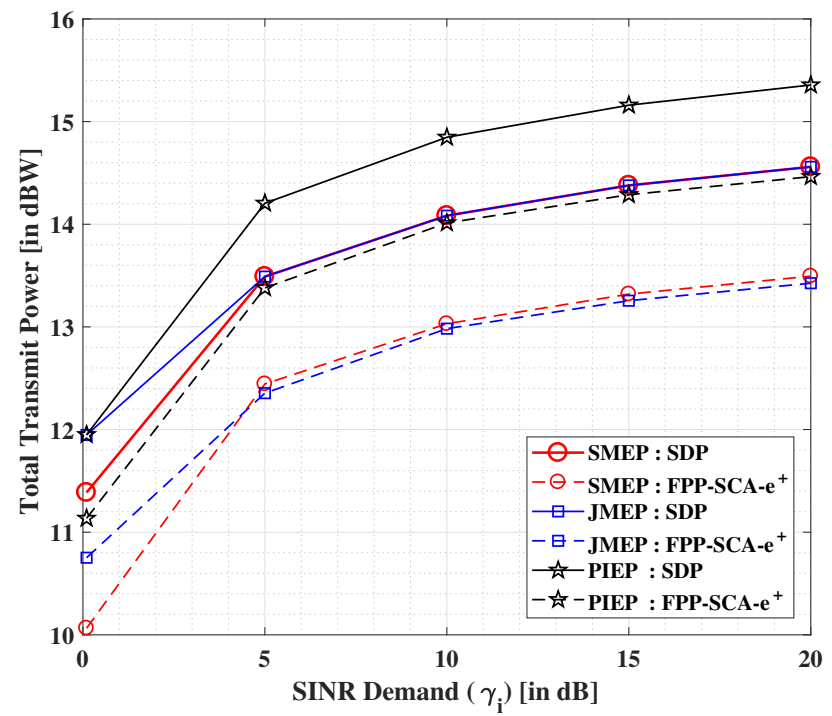

Fig. 4: Total transmit power versus the SINR demands at the users with SDP and FPP-SCA-e ${ }^{+}$techniques, where $\xi_{i}=2.5 \mu \mathrm{J}$.

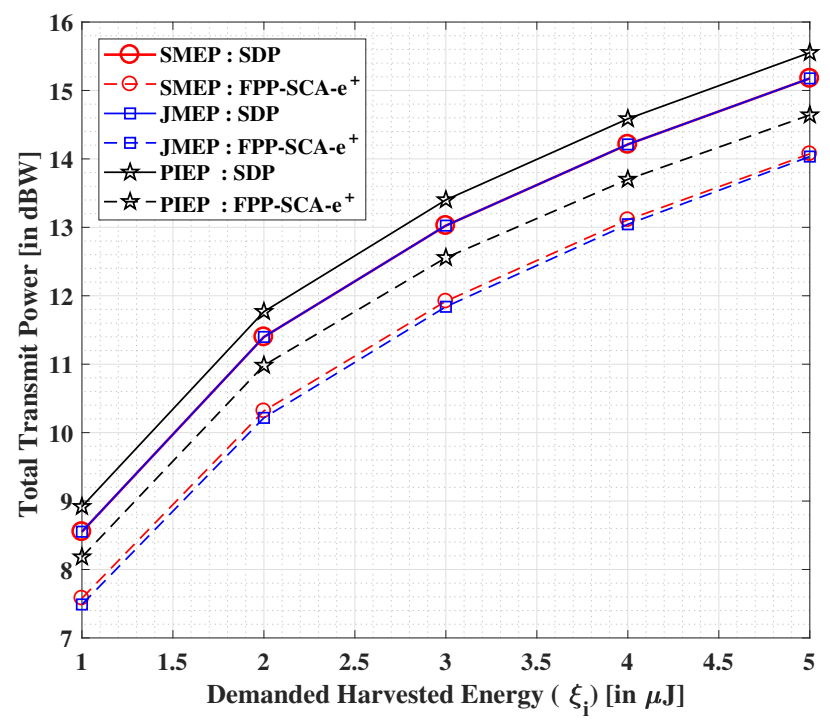

Fig. 5: Total transmit power versus the harvested energy demands at the users with SDP and FPP-SCA-e ${ }^{+}$, where $\gamma_{i}=1 \mathrm{~dB}$.

increment in case of FPP-SCA-e is around $5 \%$ to $15 \%$ (for the three scenarios).

Finally, we compare the three methods, namely, SDP, FPPSCA-e, and FPP-SCA-e ${ }^{+}$in Tables II and III, respectively for the SMEP and JMEP scenarios. We find that the solution via SDR is considered the best starting point for FPP-SCA-e, as also illustrated for similar method in [12]. Hence, we consider only SDP and FPP-SCA-e ${ }^{+}$methods for further analysis.

\section{Experimental Findings}

This sub-section presents the results obtained via numerical experiments. In comparison with [11] and [12], we consider a more practical channel model whereas the problem formulated in this work involves an additional $\mathrm{EH}$ constraint. Based on the experimental findings, we set $\lambda=10^{12}$ for FPP-SCA-e ${ }^{+}$, and $\lambda=10^{15}$ for FPP-SCA-e. These values are chosen to ensure the

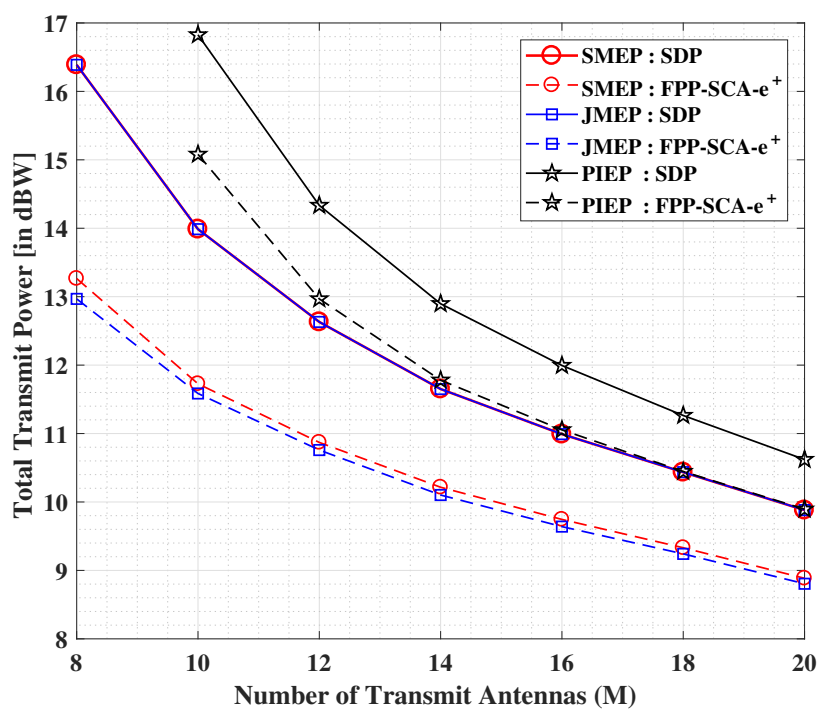

Fig. 6: Total transmit power versus number of antennas, using SDP and FPP-SCA-e ${ }^{+}$techniques, with $\gamma_{i}=5 \mathrm{~dB}$ and $\xi_{i}=1 \mu \mathrm{J}$.

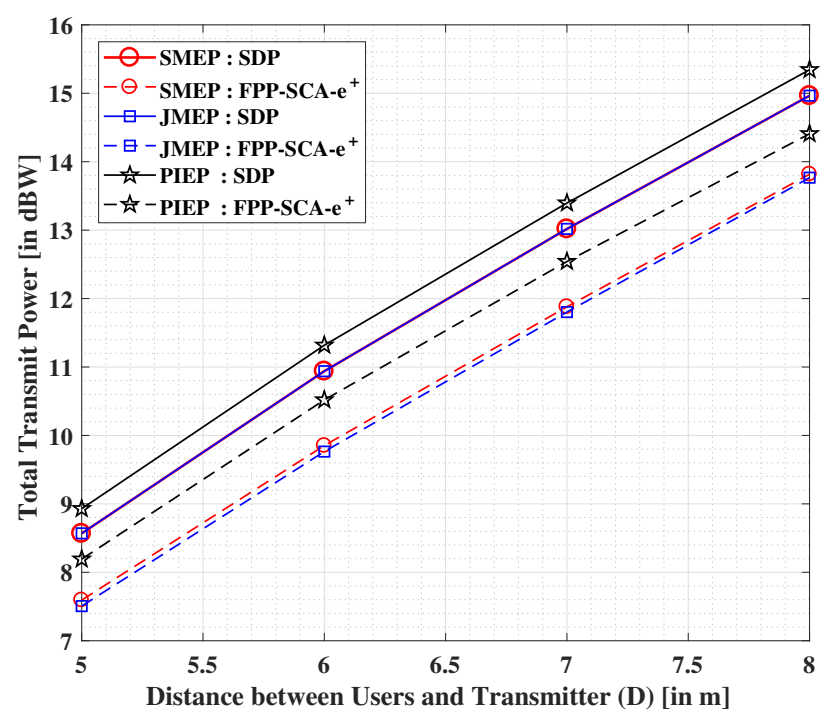

Fig. 7: Total transmit power versus the distance between the users and transmitter, with $\gamma_{i}=1 \mathrm{~dB}$ and $\xi_{i}=1 \mu \mathrm{J}$.

feasibility of considered problem in $(P 4)$, and to force the slack variables in $\mathbf{v}$ towards zero. Specifically, with the considered channel model, and given that $\gamma_{i}$ and $\xi_{i}$ are both non-zeros, we propose to choose $\lambda \geq 1 /\left(\min \left(\gamma_{i} / 1 \mathrm{~dB}, \xi_{i} / 1 \mathrm{~J}\right)\right)^{2}$.

In Fig. 4, we investigate the performances of the three scenarios, namely, SMEP, JMEP, and PIEP, wherein the effect of increasing SINR demands on the total transmit power is examined for fixed $\xi_{i}=2.5 \mu \mathrm{J}$. We observe that the total transmit power increases with growing SINR demands, for all the three above-mentioned scenarios. In addition, the proposed FPP-SCA-e ${ }^{+}$method provides a better estimate to the total transmit power in comparison to SDP. As noticed via both SDP and FPP-SCA-e ${ }^{+}$techniques, SMEP is found to perform better than JMEP in the low-SINR regimes while JMEP shows marginal advantages over SMEP in higher SINR regions. However, both JMEP and SMEP are seen to outperform PIEP 
in terms of total transmit power optimization for increasing SINR demands.

In Fig. 5, we present the impact on the total transmit power with increasing values of EH demands, where $\gamma_{i}=1 \mathrm{~dB}$. The objective is seen to increase with growing EH demands for all the three considered scenarios, viz., SMEP, JMEP, and PIEP. The performances of SMEP and JMEP are found to be considerably better in comparison to PIEP, for both SDP and FPP-SCA-e ${ }^{+}$techniques. The optimized values of total transmit powers are nearly same for SMEP and JMEP in case of SDP. However, JMEP is observed to perform slightly better than SMEP in terms of transmit power optimization via FPP-SCA$\mathrm{e}^{+}$method, for increasing EH demands.

Fig. 6, shows the result of increasing the number of transmit antennas on the total optimized transmit power for $\gamma_{i}=$ $5 \mathrm{~dB}$ and $\xi_{i}=1 \mu \mathrm{J}$. A general decreasing trend of total transmit power is observed from both SDP and FPP-SCA-e ${ }^{+}$ techniques for growing number of antennas, with latter (FPP$\mathrm{SCA}^{-} \mathrm{e}^{+}$) providing better solutions in comparison to the former (SDP). From an overall performance perspective, both JMEP and SMEP outperforms PIEP. However, similar outcomes are obtained for JMEP and SMEP through SDP, while JMEP is found to perform marginally better than SMEP in the case of FPP-SCA- $\mathrm{e}^{+}$. This marginal effect is due to the presence of an extra precoder in SMEP over JMEP.

We depict in Fig. 7 the performance analysis of the three scenarios (SMEP, JMEP and PIEP) in terms of total transmit power against increasing distance between the transmitter and users, with $\gamma_{i}=1 \mathrm{~dB}$ and $\xi_{i}=1 \mu \mathrm{J}$. Please note that any point corresponding to $(D)$ on the $\mathrm{x}$-axis portrays the result for random arrangements of each user between $(D)$ and $(D-1)$ for each instance of the experimental realizations. The total transmit power escalates significantly with increasing values of distances between the transmitter and users. Similar as above, we find that the SMEP and JMEP systems exceed over PIEP for both SDP and FPP-SCA- $\mathrm{e}^{+}$techniques. A slender gap is observed between the performances of SMEP and JMEP in case of FPP-SCA-e ${ }^{+}$technique while both shows identical performances with SDP.

\section{CONCLUSION}

We proposed a novel technique named FPP-SCA-e, to address the problem of total transmit power minimization in three considered scenarios, namely, SMEP, JMEP, and PIEP. The developed technique was found to be suitable for solving a QCQP problem that consisted of both ID and EH users. Moreover, coexistence of heterogeneous user types was established under a practically motivated system model. Performance benefits of the FPP-SCA-e technique were shown over SDP via numerical results. From the systems perspective, SMEP was found to be most suitable candidate for practical implementation due to its flexible precoder designs and superior performance over JMEP and PIEP in the low-SINR regime. Moreover, this system may facilitate exclusive waveform designs targeting the ID and $\mathrm{EH}$ users separately, with an anticipated improvement in the overall system efficiency.

\section{ACKNOWLEDGMENT}

This work was partially supported by the Luxembourg National Research Fund (FNR) in the framework of the FNR-FNRS bilateral project - InWIP-NET : Integrated Wireless Information and Power Networks (R-AGR-0700-10-V).

\section{REFERENCES}

[1] S. Gautam, T. X. Vu, S. Chatzinotas, and B. Ottersten, "Cache-Aided Simultaneous Wireless Information and Power Transfer (SWIPT) With Relay Selection," IEEE J. Sel. Areas Commun, vol. 37, no. 1, pp. 187201, Jan 2019.

[2] Q. Shi, L. Liu, W. Xu, and R. Zhang, "Joint Transmit Beamforming and Receive Power Splitting for MISO SWIPT Systems," IEEE Trans. Wireless Commun., vol. 13, no. 6, pp. 3269-3280, June 2014.

[3] S. Gautam, E. Lagunas, S. Chatzinotas, and B. Ottersten, "Wireless Multigroup Multicast Precoding with Selective RF Energy Harvesting," in 27th European Sig. Processing Conf. (EUSIPCO), September 2019.

[4] R. Tandon, S. A. Jafar, S. Shamai, and H. V. Poor, "On the Synergistic Benefits of Alternating CSIT for the MISO Broadcast Channel," IEEE Trans. Inf. Theory, vol. 59, no. 7, pp. 4106-4128, July 2013.

[5] L. R. Varshney, "Transporting information and energy simultaneously," in IEEE Int. Symp. Inf. Theory, July 2008, pp. 1612-1616.

[6] M. R. A. Khandaker and K. Wong, "SWIPT in MISO Multicasting Systems," IEEE Wireless Commun. Lett., vol. 3, no. 3, pp. 277-280, June 2014.

[7] Z. Ding, C. Zhong, D. W. K. Ng, M. Peng, H. A. Suraweera, R. Schober, and H. V. Poor, "Application of smart antenna technologies in simultaneous wireless information and power transfer," IEEE Comm. Mag., vol. 53, no. 4, pp. 86-93, April 2015.

[8] N. D. Sidiropoulos, T. N. Davidson, and Z.-Q. Luo, "Transmit beamforming for physical-layer multicasting," IEEE Trans. Signal Process., vol. 54, no. 6, pp. 2239-2251, June 2006.

[9] M. Alodeh, D. Spano, A. Kalantari, C. G. Tsinos, D. Christopoulos, S. Chatzinotas, and B. Ottersten, "Symbol-Level and Multicast Precoding for Multiuser Multiantenna Downlink: A State-of-the-Art, Classification, and Challenges," IEEE Commun. Surveys Tuts., vol. 20, no. 3, pp. 17331757, thirdquarter 2018.

[10] Ö. T. Demir and T. E. Tuncer, "Antenna Selection and Hybrid Beamforming for Simultaneous Wireless Information and Power Transfer in Multi-Group Multicasting Systems,' IEEE Trans. Wireless Comm., vol. 15, no. 10, pp. 6948-6962, Oct 2016.

[11] O. Mehanna, K. Huang, B. Gopalakrishnan, A. Konar, and N. D. Sidiropoulos, "Feasible Point Pursuit and Successive Approximation of Non-Convex QCQPs," IEEE Signal Processing Letters, vol. 22, no. 7, pp. 804-808, July 2015.

[12] D. Christopoulos, S. Chatzinotas, and B. Ottersten, "Multicast multigroup beamforming under per-antenna power constraints," in IEEE Int. Conf. Comm. (ICC), June 2014, pp. 4704-4710.

[13] B. Clerckx and E. Bayguzina, "Waveform Design for Wireless Power Transfer," IEEE Trans. Signal Process., vol. 64, no. 23, pp. 6313-6328, 2016.

[14] J. Guo, H. Zhang, and X. Zhu, "Theoretical analysis of RF-DC conversion efficiency for class-F rectifiers," IEEE Trans. Microw. Theory Techn., vol. 62, no. 4, pp. 977-985, 2014.

[15] S. Gautam, E. Lagunas, A. Bandi, S. Chatzinotas, S. K. Sharma, T. X. Vu, S. Kisseleff, and B. Ottersten, "Multigroup Multicast Precoding for Energy Optimization in SWIPT Systems With Heterogeneous Users," IEEE Open Journal of the Communications Society, vol. 1, pp. 92-108, 2020.

[16] S. Boyd and L. Vandenberghe, Convex optimization, Cambridge university press, 2004.

[17] M. Grant and S. Boyd, "CVX: Matlab software for disciplined convex programming, version 2.1," http://cvxr.com/cvx, Mar. 2014.

[18] K. Krishnan, Linear programming (LP) approaches to semidefinite programming (SDP) problems, Ph.D. thesis, Citeseer, 2002.

[19] P. Series, "Propagation data and prediction methods for the planning of indoor radiocommunication systems and radio local area networks in the frequency range $900 \mathrm{MHz}$ to $100 \mathrm{GHz}$," Recommendation ITU-R, pp. 1238-7, 2012.

[20] S. Gautam, E. Lagunas, S. Chatzinotas, and B. Ottersten, "Relay Selection and Resource Allocation for SWIPT in Multi-User OFDMA Systems," IEEE Trans. Wireless Commun., vol. 18, no. 5, pp. 2493-2508, May 2019. 